\title{
A Two-Layer Model of the North Atlantic Thermocline
}

\author{
P. P. Nimler, E. Simco and R. Larue \\ Physical Oceanography Laboratory, Nova University, Fl. Landerdale, Fla.
}

(Manuscript received 12 May 1971, in revised form 12 June 1971)

\section{ABSTRACT}

\begin{abstract}
A two-layer model of a mid-oceanic thermocline is developed, and a comparison of the depth and temperature of the thermocline in this model is made with a root-mean-square description of the hydrographic structure of the North Atlantic. It is shown that the Sverdrup balance is maintained within the rms errors, and the entire estimated heat flux from the atmosphere is used to produce the observed density changes along the path of persistent flow.
\end{abstract}

\section{Introduction}

In this paper we develop the simplest two-layer model of the persistent, mid-oceanic thermocline and discuss its relevance to the observed structure in the subtropical North Atlantic. In this model, the density of the warmer surface water, as well as its depth, is a function of the longitude and latitude, and the horizontal contrasts of the structure of the layer across the ocean are related to the sea-surface heating and wind stress. The quiescent abyssal water mass is of constant density throughout. The Sverdrup balance in this model is tested by observations in the area of the North Atlantic which extends over $20-60 \mathrm{~W}$ longitude and $8-36 \mathrm{~N}$ latitude. A two-dimensional, least-square rms distribution of the two-layer integrated pressure field is computed from the hydrographic observations (from 704 NODC stations which extend below $2000 \mathrm{~m}$ ), and this distribution is shown to be remarkably consistent with the structure that can be maintained by the observed wind stress over the same region (Hellerman, 1967). Also, the heating rate which is required to maintain the observed temperature contrast is computed from the rms fit to the observations. The latter computation reveals that nearly all the estimated heat flux into the ocean (Budyko, 1956) is required to maintain the observed horizontal temperature (density) contrasts along the path of persistent flow.

\section{The model}

Let the depth and density of the heavy water column be $D_{0}$ and $\rho_{0}$ (both assumed constant) and let the depth and density of the thinner and lighter surface water column be $D_{1}(x, y)$ and $\rho_{1}(x, y)$. In this two-layer model the velocity in the surface layer is a function of depth $z$; also $\rho_{1}$ and $D_{1}$ are functions of the latitude $y$ and longitude $x$ [for a discussion of the circulation problem in which $\rho_{1}$ is a constant, see Fofonoff (1962) and Welander (1968)]. Both $D_{1}$ and $\rho_{1}$ are the dependent variables of this problem and are determined by the dynamics and thermodynamics of the wind-driven and heated two-layer circulation. ${ }^{1}$ The circulation is driven by a surface wind stress $\tau_{0}(x, y)$, and a buoyancy flux, $g_{\alpha} \dot{Q}_{0}(x, y)$, on the ocean surface, where $\alpha$ is the thermometric expansion coefficient of sea water and $\dot{Q}_{0}$ the surface heat flux. In this model, salt is a conserved quantity.

We wish to model the dynamics of the central portion of the North Atlantic, which is bounded to the north and west by the Gulf Stream and the region where intense cold-water eddies have been observed, and to the south by the $8 \mathrm{~N}$ latitude circle and the North Atlantic Countercurrent. The hydrostatic equilibrium and the momentum balance for the upper layer are described by

$$
\rho_{0} \mathbf{f} \times \mathbf{v}_{1}=-\nabla P_{1}+\frac{\partial \tau}{\partial z}-\mathbf{k} g \rho_{1} .
$$

The usual Boussinesq and $\beta$-plane approximation is adopted in which $P$ is the pressure, $f$ the Coriolis parameter where $\mathbf{f}=\left[0,0, f_{0}+\beta y\right], \mathbf{v}=[u, v, 0]$ is the horizontal velocity vector, $\mathbf{k} g$ is the gravitational accel. eration in the vertical direction, and $z$ is the elevation relative to a MSL datum.

In the subtropical North Atlantic, the deep water is of relatively homogeneous density and comprises a much thicker layer $(\sim 3500 \mathrm{~m})$ than the warmer surface water $(\sim 500 \mathrm{~m})$ in which the predominant horizontal and vertical density gradients are found. Since the

\footnotetext{
${ }^{1}$ Kamenkovich (1969) has suggested a very similar approach in the development of a model of the horizontal temperature structure of the surface layers of the ocean, and Wyrtki and Haberland (1968) have used the thermodynamics, but not the dynamics, of a two-layer model in a discussion of the lateral distribution of heat in the North Pacific Ocean. Yoshida (1965) also employs a two-parameter description of the density field in the North Pacific Ocean, but relates his computations only qualitatively to the observations and restricts his analysis to the case $\dot{Q}_{0}=0$.
} 
transport of water in the bottom layer is not expected to be appreciably greater than in the surface layer, the velocity of the column of water in the abyss is much smaller, $\mathrm{O}\left(D_{1} / D_{0}\right)$, than in the surface layer. The vertical component of (1) can be integrated as if the lower layer were at rest; the result, for the upper layer, with $\Delta=\rho_{0}-\rho_{1}$, is

$$
P_{1}=P_{0}+\left\{-\rho_{0} g z+\Delta g\left[z+D_{1}\right]\right\}\left\{1+\mathrm{O}\left(D_{1} / D_{0}\right)\right\},
$$

where $P_{0}$ is sea level atmospheric pressure (assumed constant).

The conservation of mass in the moving laver is most simply expressed as

$$
\nabla \cdot \int_{-D_{1}}^{0} \mathbf{v}_{1} d z=0
$$

In the derivation of (3), upwelling from the abyss has not been taken into account. The most recent estimates of mid-ocean upwelling (Gates, 1969; Rooth, 1970² Veronis, 1969) show that Ekman divergence, or the order of the horizontal divergence of (3), is the order of $10^{-4} \mathrm{~cm} \mathrm{sec}^{-1}$, but the abyssal upwelling is a much slower flow, of the order of $10^{-5} \mathrm{~cm} \mathrm{sec}^{-1}$.

Eqs. (1), (2) and (3) with the conditions $\tau=\tau_{0}$ at the sea surface and $\tau=0$ at the interface lead to the Sverdrup equation for the integrated pressure fields [Sverdrup, 1947; see Robinson (1965) for the case $\Delta=$ constant]:

$$
\frac{\partial}{\partial x}\left[\frac{g}{2} \Delta D_{1}^{2}\right]=\frac{f^{2}}{\beta} \mathbf{k} \cdot \nabla \times\left[\frac{\tau_{0}}{f}\right] .
$$

Eq. (4) is used to determine $D_{1}$ when $\Delta$ is given; the quantity $(g / 2) \Delta D_{1}{ }^{2}$ in this model is simply the integrated pressure anomaly.

The density variation, or $\Delta$, is determined from the second law of thermodynamics. Let $\rho_{0}-\rho_{1}=\alpha\left[T_{1}-T_{0}\right]$ $-\beta_{0}\left[S_{1}-S_{0}\right]$, where $S$ is the salinity of the water and $\beta_{0}=(\partial \rho / \partial S)_{T}$. Since only the surface layer is heated, $S_{1}$ is conserved and $\rho_{0}$ is a constant; the conservation laws for heat and salt are written as

$$
\mathbf{v}_{1} \cdot \nabla \rho_{0}=0, \quad \mathbf{v}_{1} \cdot \nabla T_{1}=-\frac{\partial \dot{Q}}{\partial z}, \quad \mathbf{v}_{1} \cdot \nabla S_{1}=0
$$

Multiply the second part of (5) by $\alpha$ and the third part by $\beta_{0}$ and subtract from the first part. The vertical integral of this sum is (Niiler and Doubleday, 1970)

$$
\int_{-D_{1}}^{0} \mathbf{v}_{1} \cdot \nabla(\Delta) d z=\alpha \dot{Q_{0}}(x, y)
$$

In the derivation of (6) the heat flux into the abyss has been neglected as has the abyssal mass flux in (3).

\footnotetext{
2 Rooth, C., private communication.
}

An alternate definition for $\dot{Q}_{0}$ in (6) is the rate at which the ocean surface is heated minus the rate at which heat is supplied to the abyss. The simplest theories of the diffusive and continuously stratified thermocline indicate [see Veronis (1969) for a review] that heat diffuses into the abyss at a rate proportional to the vertical diffusivity $k$ and the temperature (density) difference $\Delta$ between the surface layers and the abyss i.e., $\propto k \Delta$. In these models there also is an upwelling of a cold mass of water at the bottom of the thermocline which is proportional to $k \Delta$, and this cold upwelling essentially balances the diffusion of heat down from the surface. The model we discuss here is one in which $k$ is small $[O(1)$, in cgs units], and the effects of both abyssal upwelling as well as heat flux to the abyss are neglected in the dynamics of the upper layer. However as shown by Stommel and Arons (1960) and Veronis (1969), this upwelling is a crucial constraint in the dynamics of the more sluggish abyssal circulation.

Considerable simplification of the apparent nonlinear equation for $\Delta$ results in the elimination of $\mathbf{v}_{1}$ and $D_{1}$ from (6). We begin by integrating (4) to obtain

$$
\frac{g}{2} \Delta D_{1}{ }^{2}=\frac{1}{2} G(y)+\int_{L}^{x}\left\{\frac{f^{2}}{\beta} \mathbf{k} \cdot \nabla \times\left(\frac{\tau_{0}}{f}\right)\right\} d x .
$$

In (7), $G(y)$ is the value of $g \Delta D_{1}{ }^{2}$ at $x=L$, and the gradient of this function is proportional to the free geostrophic zonal transport in the basin. The first boundary condition of this problem is the specification of $G$.

Secondly, it is convenient to define a mass transport streamfunction which exists in view of (3):

$$
\rho_{0} \int_{-D_{1}}^{0} \mathrm{v}_{1} d \dot{z}=\mathrm{k} \times \nabla \Psi
$$

To obtain the equations for $\Psi$, substitute (8) into the vertical integral "of the $x, y$ components of (1), whereby

$$
\left.\begin{array}{c}
f \frac{\partial \Psi}{\partial x}=\frac{1}{2} \frac{\partial}{\partial x}\left[g \Delta D_{1}^{2}\right]-\tau_{0}{ }^{(x)} \\
\frac{\partial \Psi}{\partial y}=\frac{1}{2} \frac{\partial}{\partial y}\left[g \Delta D_{1}^{2}\right]-\tau_{0}{ }^{(y)}
\end{array}\right\}
$$

The solution for $\Psi$ is obtained by eliminating $\Delta D_{1}{ }^{2}$ from (9) and integrating the subsequent expression in $x$; this yields

$$
\Psi=\Psi_{0}(y)+\frac{1}{\beta} \int_{L}^{x} \mathbf{k} \cdot \nabla \times \tau_{0} d x
$$

where $\Psi_{0}$ is the free geostrophic transport at the longitude $x=L$, and

$$
\Psi_{0}=\int_{0}^{y} \frac{1 d G}{f d y} d y-\int_{0}^{y}\left[\frac{\tau_{0}^{(y)}}{f}\right]_{x=L} d y .
$$


The Sverdrup transport in the basin is the sum of the geostrophic and Ekman transports, and, when $\Delta D_{1}{ }^{2}$ and $\tau_{0}$ are given, is obtained from

$$
\begin{aligned}
f \Psi=\frac{g}{2} \Delta D_{1}^{2}+f \int_{0}^{y}\left(\frac{\beta}{f^{2}} G\right) d y & -\int_{0}^{y}\left[\frac{\tau_{0}^{(y)}}{f}\right]_{x=L} d y \\
& -\int_{L}^{x} \tau_{0}^{(x)} d x-\frac{1}{2} G^{(0)} .
\end{aligned}
$$

The lines of constant $\Psi$ are the path lines of the total transport vector in the moving layer, and this function is completely specified by the wind-stress distribution and the free geostrophic flux $\Psi_{0}$. The vertical structure of the velocity field, below the Ekman layer, is functionally related to the heating rate at the ocean surface. The geostrophic and z-dependent (baroclinic) part, however, does not contribute to the net advection on the left-hand side of (6).

With $\Psi$ known, (6) becomes a linear, first-order, partial differential equation for $\Delta$, i.e.,

$$
\frac{\partial \Psi}{\partial x} \frac{\partial \Delta}{\partial y}-\frac{\partial \Psi}{\partial y} \frac{\partial \Delta}{\partial x}=\rho_{0} a \dot{Q}_{0} .
$$

Eq. (13) states that following the path of the warm column of water, the water column gets lighter $(\Delta$ increases) if there is a net flux of heat into the ocean, and heavier ( $\Delta$ decreases) if heat is extracted from the layer. Since lines of constant total transport are the characteristics of (13), a unique solution requires $\Delta$ be specified (say $\Delta=\Delta_{\infty}$ ) on a line which intersects the path lines of the total transport. In other words, the density of the water column which flows into the region has to be specified, and (13) describes the horizontal contrasts which are produced in $\Delta$ along the path of flow. [Recall that a gyre cannot be completed with purely geostrophic flow (Stommel, 1965).] The boundary conditions for the geostrophic part of the basin are the functions $G$ (or $\Psi_{0}$ ) and $\Delta_{\infty}$. The solution to (13) is obtained by using $y$ and $\Psi$ as independent coordinates and integrating in $y$ along lines of fixed $\Psi$ :

$$
\Delta=\Delta_{\infty}\left(\Psi_{\infty}\right)+\int_{y_{\infty}}^{y} \frac{a \dot{Q}_{0} \beta}{\left[\mathbf{k} \cdot \nabla \times \tau_{0}\right]} d y_{\Psi} .
$$

In this integral, $\Delta_{\infty}\left(\Psi_{\infty}\right)$ is the inlet condition for the density, and $y_{\infty}$ describes the location of the initial curve along which $\Delta_{\infty}$ is specified.

We now compare the prediction of this model with the North Atlantic hydrographic structure by using (14) to compute the changes of $\Delta$ from the initial values, and subsequently determine $D_{1}$ from (7); $\Delta_{\infty}$ is chosen as the density of the surface layer at the $36 \mathrm{~N}$, and $G$ is computed from the hydrographic data at $20 \mathrm{~W}$. Initially, smooth analytic functions are specified for $\dot{Q}_{0}$ and

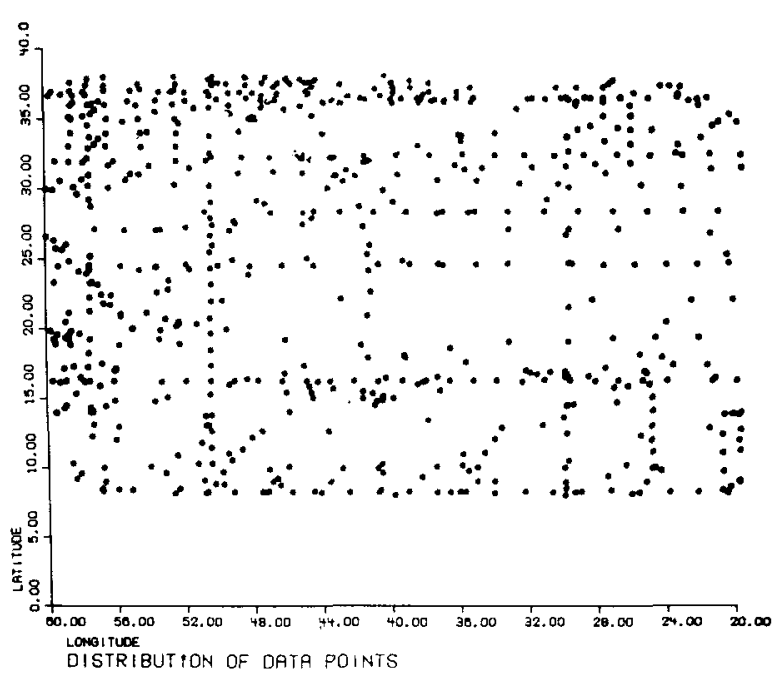

Fig. 1. Distribution of NODC hydrographic stations which are used in the least rms polynomial description of the depth and density of the warm-water circulation.

$\mathbf{k} \cdot \nabla \times \tau_{0}$ in the numerical integration of (14). Finally, the observed distributions of $\Delta$ and $D_{1}$ are used to compute the required $\dot{Q}_{0}$ from (12) and (13).

\section{The two-layer model of the North Atlantic}

\section{a. Hydrographic data}

Fig. 1 shows the distribution of the NODC hydrographic stations in the area $20-60 \mathrm{~W}$ and $8-36 \mathrm{~N}$ which are used to compute the observed values of $\Delta$ and $D_{1}$. The abyssal region is identified with the water column of horizontally invariant, vertically averaged density above a fixed deep level. The average density of the remaining water column to the ocean surface is identified with that of the more rapidly moving warmer laver. In the vertical integrations of the density field, a cubic spline fit to $\sigma_{i}$ is made between the hydrographic bottle data samples.

Each hydrographic cast record was visually checked for errors on the NODC magnetic tape and for consistency with adjoining data in a similar geographic region. Only those hydrographic casts which had regularly spaced bottle data to 2000 or $3000 \mathrm{~m}$ were used. In this way, 479 station records were obtained which extend below $3000 \mathrm{~m}$ and 704 which extend below $2000 \mathrm{~m}$.

The computation began by a numerical integration of the measured density profile from a fixed deep level $\left(2000\right.$ or $3000 \mathrm{~m}$ ) until the average $\sigma_{t}$ approached a fixed predetermined value of $\sigma_{t_{0}}$ at a depth $z_{0}, D_{1}$ being set equal to the depth of the remaining water column from $z_{0}$ to the ocean surface. The numerical integration of the hydrographic cast continued in $z$ to the ocean surface and $\sigma_{t_{1}}$ was set equal to the average value of the $\sigma_{t}$ of the water column from $z_{0}$ to the ocean surface; 
TABLE 1. Summary of the rms two-layer description of the North Atlantic hydrographic structure. $\partial_{x}$ is the total longitudinal contrast and $\partial_{y}$ is the latitudinal contrast across the basin.

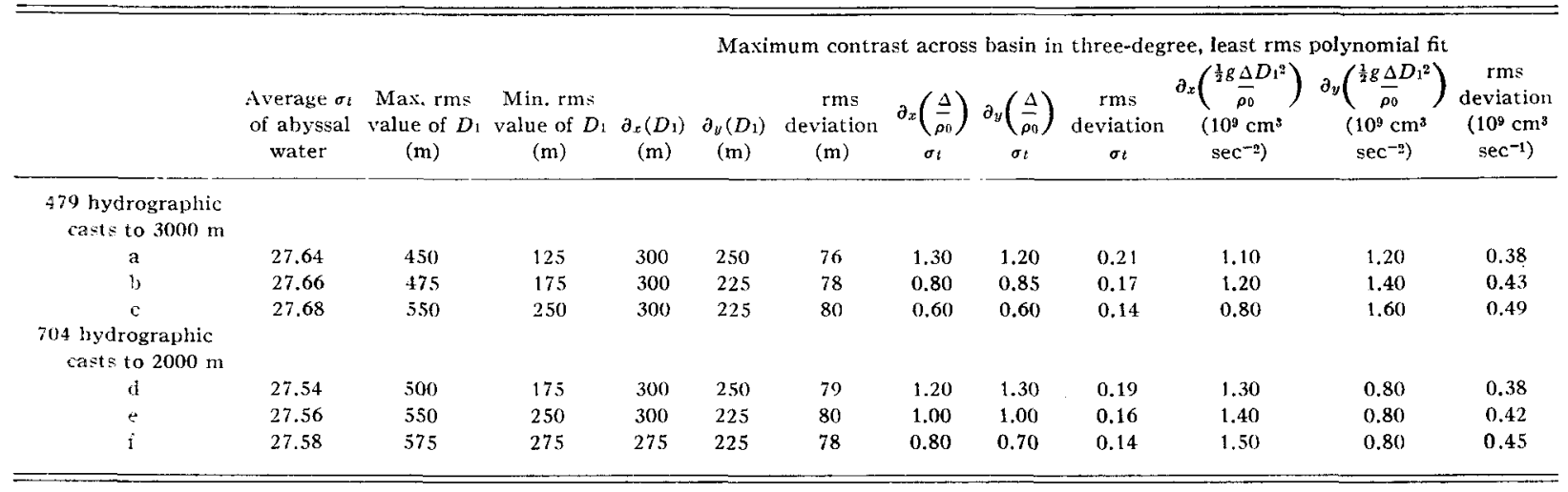

for the purpose of correlating the model with observations, $\Delta \equiv \sigma_{t_{0}}-\sigma_{t_{1}}$.

Individual calculations for $D_{1}, \Delta$ and $\frac{1}{2} g \Delta D_{1}{ }^{2}$ were carried out for the entire set of hydrographic records, and a two-dimensional polynomial fit obtained for each of the distributions. The results of this analysis are summarized in Table 1. Practically no successively smaller rms deviations were obtained with a polynomial of higher than three degrees, and since the computer plotting routine contours $D_{1}$ to the nearest $25 \mathrm{~m}$ and $\Delta$ to $0.10 \sigma_{\iota}$ unit, the horizontal contrasts in $D_{1}, \Delta$ and $\frac{1}{2} g \Delta D_{1}{ }^{2}$ are tabulated in these units for a three-degree polynomial fit.

The simple theory which is outlined in the first part of this paper predicts only the changes of $\Delta$ and $\frac{1}{2} g \Delta D_{1}{ }^{2}$ across the basin, and not their absolute values. It is seen that the test of the model can be carried out quite adequately within the uncertainties introduced by the random character of the data and the particular rms description employed, in that the total observed contrasts across the basin were larger than the rms errors. The best description of the data is obtained when the abyssal column is of $27.64 \sigma_{t}$ (from $3000 \mathrm{~m}$ ) or $27.54 \sigma_{t}$

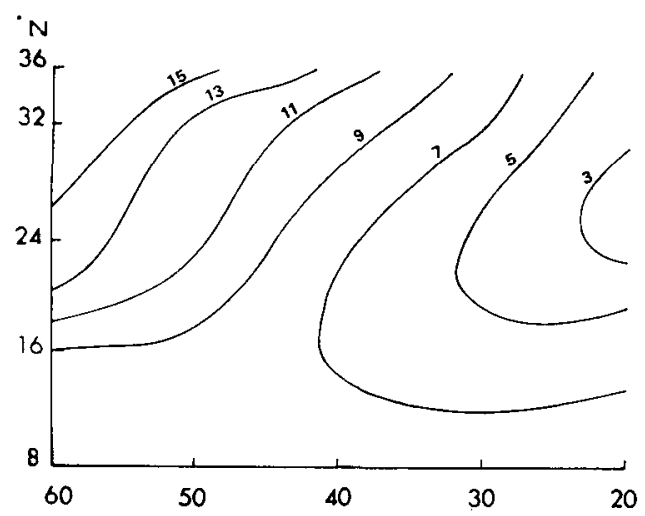

Fic. 2a. Vertically integrated pressure distribution (contours, $10^{8} \mathrm{dyn}^{-1}$ ) which can be maintained by the surface wind stress as reported by Hellerman (1967); $G[$ Eq. (7)] is derived from data in Fig. 2b. (from $2000 \mathrm{~m}$ ). Different choices of bottom-layer density basically do not affect the two-layer description of this region of the ocean, in that the horizontal contrasts of the dynamical variables in each description differs less than the computed rms uncertainties in each description.

\section{$b$. The Sverdrup relationship}

The simplest test of the model is the comparison of the observed integrated pressure map (the distribution of $\frac{1}{2} g \Delta D_{1}{ }^{2}$ ) with the distribution predicted by the Sverdrup relation (7). The wind-stress distribution reported by Hellerman (1967) is used to calculate the Ekman divergence and a numerical integration of (7) produces Fig. 2a. The observed rms distribution of the integrated pressure is displayed in Fig. $2 b$, for the best rms fit to the hydrographic data. The agreement is everywhere within the rms uncertainties. We can conclude that in this region of the North Atlantic the Sverdrup balance is maintained, and this balance can be adequately described within the context of a two-layer circulation model.



Fig. 2b. The observed vertically integrated pressure distribution in a two-layer model, in which the quiescent abyssal water mass above $2000 \mathrm{~m}$ is at an average density of $27.54 \sigma_{t}$. The hydrographic data, which is depicted in Fig. 1, has been fitted by a three-degree, two-dimensional polynomial, and contours of $\frac{1}{2} g \Delta D_{1}^{2}$ are in units of $10^{8}$ dyn $\mathrm{cm}^{-1}$; the rms deviation is $3.8 \times 10^{8}$ dyn $\mathrm{cm}^{-1}$. 


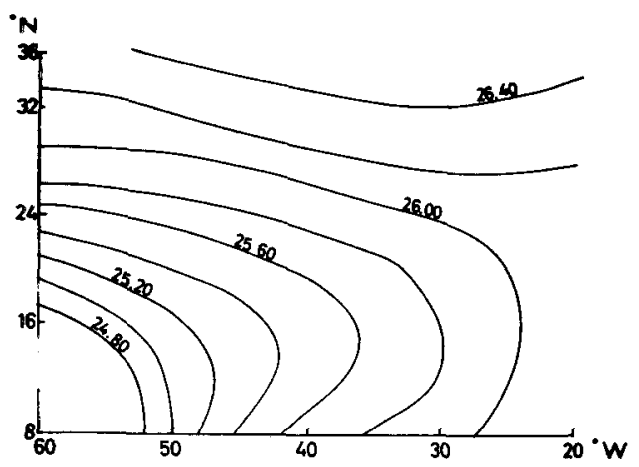

IFIG. 3. The distribution of the observed surface $\sigma_{t_{1}}$ of the upper layer, which corresponds to the representation in Fig. $2 \mathrm{~b}$; the rms deviation is $0.19 \sigma_{l}$.

The application of the same description in the region which is north and west of Bermuda (or basically the Sargasso Sea) must yield considerable discrepancies. The fundamental reason for this conjecture is that the observed total warm-water transport in the Gulf Stream system is at least three times as great as the transport which can be obtained from Sverdrup theory [Eq. (10)]. The maximum value of

$$
\left\{\int_{L}^{x_{0}} \mathbf{k} \cdot \nabla \times \tau_{0} d x\right\}
$$

in the North Atlantic is $22.5 \times 10^{6} \mathrm{~m}^{3} \mathrm{sec}^{-1}$ [computation by Hellerman (1965) with same data as used here], while the observed maximum values of the directly measured warm-water transport in the Gulf Stream system, at the same latitude, exceeds $60 \times 10^{6} \mathrm{~m}^{3} \mathrm{sec}^{-1}$ (Richardson et al. 1969). It is quite probable that a strong geostrophic recirculation of vorticity predominates there (large $f \Psi_{0}$ ), as is indicated by the observations of the movement of strong cold-water eddies (Fuglister, 1967; Worthington, 1965), and the vorticity balance is modified by Reynolds stresses or advective terms. Finally, we note that, in the circulation pattern which appears in the bottom-frictional, homogeneous circulation model when the frictional parameter is chosen such that the computed maximum transport is equal to the observed maximum transport, the entire northern and western region of the Gulf Stream is strongly nonlinear. The Sverdrup balance is maintained only in the southeastern portion of the gyre (Veronis, 1966).

\section{c. The thermocline depth and density}

In Figs. 3 and $4 \mathrm{a}$ the observed contours of $\sigma_{t_{1}}$ and $D_{1}$ are displayed for the best $\mathrm{rms}$ fit. The region of colder (dense) water along the $20 \mathrm{~W}$ meridian reflects the cold-water mass along the west African coast. The strong longitudinal density contrast is not found in the surface density distribution, except in the late summer, but since $\Delta$ is the average density of the thermocline,

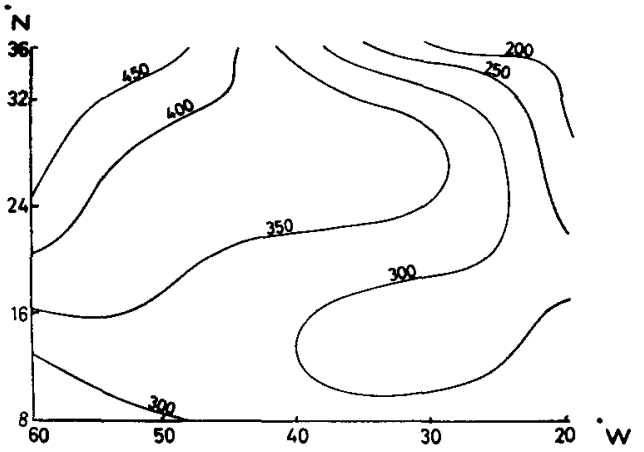

FIG. 4a. The distribution of the observed depth of the surface layer $D_{1}(\mathrm{~m})$ which corresponds to the representation in $F i g .2 \mathrm{~b}$; the rms deviation is $79 \mathrm{~m}$.

the deeper longitudinal temperature contrast is reflected in the present distribution. The shallow region of the thermocline at $30 \mathrm{~W}, 16 \mathrm{~N}$ is related directly to the observation that, at this location, $\mathbf{k} \cdot \nabla \times\left(\boldsymbol{\tau}_{0} / f\right)>0$, so the wind stress drives a divergent Ekman transport and there is a compensating upwelling. This simple description does not afford an explanation for the deepening of the warm layer at $25 \mathrm{~N}, 40 \mathrm{~W}$ and subsequent shallowing at $36 \mathrm{~N}, 30 \mathrm{~W}$. For contrast, we present a twodegree, two-dimensional polynomial fit to $D_{1}$ in Fig. 4 b, in which it is seen that the features of the shallow area at $30 \mathrm{~W}, 16 \mathrm{~N}$ are preserved while the latter are more salient features of the circulation pattern and do not appear on the map. No significant difference in the distribution of $\Delta$ can be obtained with a two-degree polynomial fit to the data, and we have not included this in the presentation.

\section{d. Integration of the analytic model and the computed heating rate}

The integration of the equations for $\Delta$ and $D_{1}$ are straightforward. In the integration of (14) we specify: 1) the $\beta$ plane to be centered at $32 \mathrm{~N} ; 2)$ a wind-stress curl $\left[\mathbf{k} \cdot \nabla \times \tau_{0}\right]$ which varies sinusoidally with latitude,

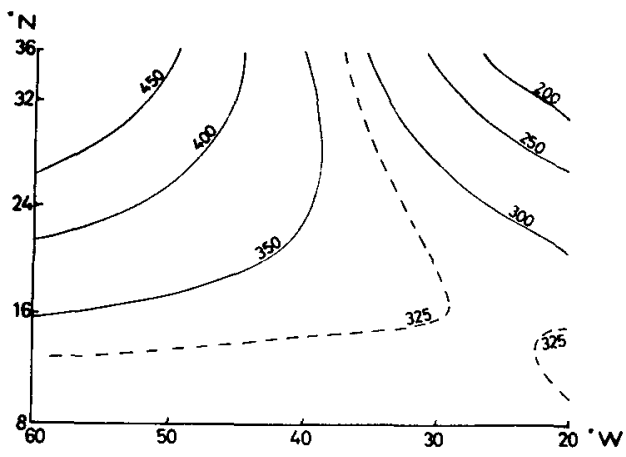

FIG. $4 \mathrm{~b}$. The distribution of the observed depth of the surface layer $D_{1}(\mathrm{~m})$. The representation is identical to Fig. $4 \mathrm{a}$, except that a second-order, two-dimensional polynomial fit is contoured; the $\mathrm{rms}$ deviation is $83 \mathrm{~m}$. 


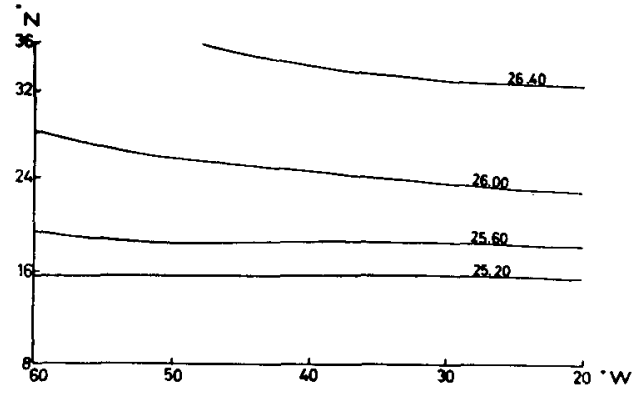

Fic. 5. The distribution of $\sigma_{t_{1}}$ for the integration of (14). See text for the values of the physical parameters.

which has a maximum value of $10^{-8} \mathrm{dyn}^{-3}$ at $32 \mathrm{~N}$, and which changes sign at $8 \mathrm{~N} ; 3$ ) a sinusoidally varying surface heating which has a maximum value of 125 $\times 10^{-5} \mathrm{cal} \mathrm{cm}^{-2} \mathrm{sec}^{-1}$ at $8 \mathrm{~N}$ and the ocean is cooled above $32 \mathrm{~N}$; and 4) that the initial conditions for the integration, $\Delta_{\infty}, G$, correspond to the hydrographic data summarized in Table $1 \mathrm{~d}$.

A comparison of the observed distributions for $\sigma_{t_{1}}$ and $D_{1}$ (Figs. 3 and 4 ) and with the calculated distributions (Figs. 5 and 6) reveals a good agreement in the latitudinal contrast. The principal difference is the numerical difference in the value of $\partial \Delta / \partial x$, which is smaller in the analytic calculations than in the observations. In this model the density field primarily depends upon the horizontal distribution of the surface heating, and the longitudinal gradient of $\Delta$ is more directly related to the east-west assymetry of $\dot{Q}_{0}$. The rms description of $\frac{1}{2} g \Delta D_{1}{ }^{2}$ and the observations of $\tau_{0}$ (Hellerman, 1967) are used to compute the density flux divergence from the left-hand side of (13). A local expansion coefficient, $\alpha\left(T_{1}, S_{1}\right)$, as a function of the rms temperature and salinity of the surface column, is used to relate this to the heat flux divergence. The result of this calculation is shown in Fig. 7. The mean value of $\dot{Q}_{0}$ is found to be somewhat larger, but principally in line with the reported calculations of the seasonal net heat flux divergence based on the latent and sensible heat transfer and the solar radiation balance at the ocean surface. Our mean value is $38 \times 10^{-5} \mathrm{cal} \mathrm{cm}^{-2} \mathrm{sec}^{-1}$ (rms error of $33 \%$ ), while, for example, Budyko's

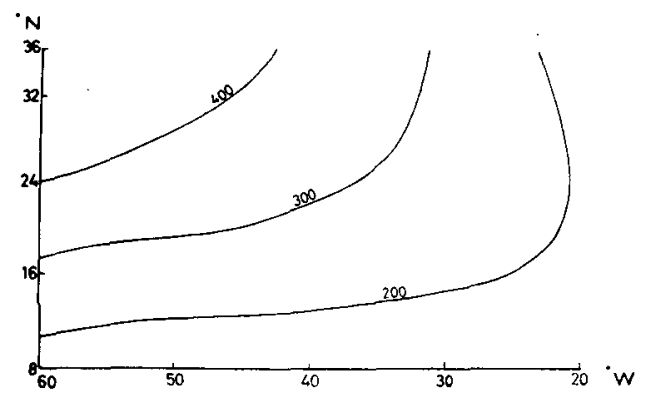

VIG. 6. The distribution of $D_{1}$ which is derived from (7) and (14). See text for the values of the physical parameters. estimate for the same region is $45 \times 10^{-5} \mathrm{cal} \mathrm{cm}^{-2} \mathrm{sec}^{-1}$ (no error available). Considerable difference, however, is noted in the details. The latter calculation suggests that stronger heating should take place in the southeast portion of this region, while our calculation shows the region of strongest heating to be in the southwest. This difference is accentuated further when salt flux due to evaporation is added in the dry trade-wind zone. The comparison of the integrated pressure map with the $\Delta$ distribution reveals that the water column which carries most of the heat $\left(\left|\tau_{0}\right|<\left|\nabla \frac{1}{2} g \Delta D_{1}^{2}\right|\right)$ moves in the direction of increasing temperature (direction of increasing $\Delta$ ) and there must be an influx of heat into the ocean over the entire region. Our estimate is a lower bound, for the cold abyssal upwelling also competes with the atmospheric heat source. Nevertheless, it is seen that there is sufficient warming of the water column in the thermocline along the path of flow to require the entire estimated net heat supply from the atmosphere.

\section{Summary}

In this short and relatively simple calculation we have developed the simplest model of the mid-oceanic thermocline and have been able to demonstrate its relevance to the problem of mid-oceanic circulation in the subtropical North Atlantic. The persistent gross structure of the North Atlantic subtropical region reflects that, within the framework of a two-layer model, the Sverdrup balance is maintained and nearly all the available heat supply from the atmosphere goes to heating the circulation above the main thermocline. A two-layer description of the dynamics and thermodynamics of the open-ocean circulation is certainly relevant and one could now pursue with some confidence the proper closure of the gyre (via boundary currents, etc.) within such a two-layer framework, because the simplest part of the broad circulation has been parameterized in a realistic manner. There is a persistent circulation pattern and density structure to the North Atlantic,

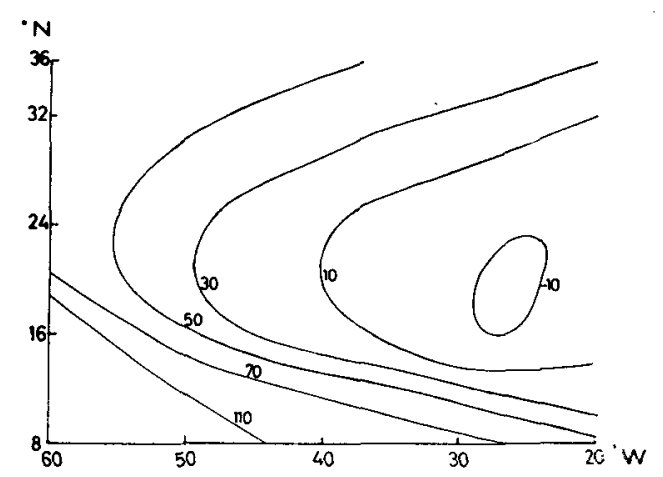

FIG. 7. The contours of $\dot{Q}_{0}\left(10^{-5} \mathrm{cal} \mathrm{cm}^{-2} \mathrm{sec}^{-1}\right)$ computed from the two-layer model of the hydrographic data, inserted on the left-hand side of (13). In this calculation, $\Delta$ is based on the polynomial description employed in Fig. $3, \frac{1}{2} g \Delta D_{1}{ }^{2}$ in Fig. $2 \mathrm{~b}$, and $\tau_{0}$ is obtained from Hellerman's $(1967)$ wind stress tables. 
whence the vorticity introduced to the ocean by the wind in this broad region of the basin must be transferred eventually to the ocean bottom, and the heat input must be transferred back to the atmosphere or transferred to adjacent basins via abyssal circulation. The regions of the basin where these processes take place are presumably in swift currents such as the Gulf Stream and the Guinea Current which ride on the western and eastern continental rise. The details of the dynamical processes of how this is accomplished still remain the central problems in the theory of the general circulation of the oceans.

Acknowledgments. The authors are grateful to the Office of Naval Research and the National Science Foundation for support during the tenure of this work. NSF Grant GA 14688, with Nova University, provided the research assistantships. The authors are also grateful to Dr. W. Powers for assistance in developing the general computing routines used in this paper.

\section{REFERENCES}

Budyko, M. I., 1956: The Heat Balance of the Earth's Surface. Translated by $N$. A. Stepanova, U. S. Weather Bureau, Washington, D. C., 1958.

Fofonoff, N., 1962 : Dynamics of ocean currents. The Sea, Chap. 3, New York, Interscience $864 \mathrm{pp}$.

Finglister, F. C., 1967: Cyclonic eddies formed by meanders of the Gulf Stream. Trans. Amer. Geophys. Union, 48, p. 123 (abstract).

Gates, W. L., 1969: The Ekman vertical velocity in any enclosed $\beta$-plane ocean. J. Marine Res. 27, 99-120.
Hellerman, S., 1965: Computation of wind stress fields over the North Atlantic Ocean. Mon. Wea. Rev., 23, 239-244.

- 1967: An updated estimate of the wind stress on the world oceans. Mon. Wea. Rev., 95, 607-626.

Kamenkovich, V. M., 1969: A model for determining the surface temperature of the ocean. Oceanology, 9, p. 28 (English translation).

Kuo, H., and G. Veronis, 1970 : Distribution of tracers in the deep oceans of the world. Deep Sea Res., 17, 29-46.

Niiler, P. P., and D. Doubleday 1970: Circulation in a windswept and cooled ocean. J. Marine Res., 28, 135-149

Richardson, W. S., W. J. Schmitz and P. P. Niiler, 1969: The velocity structure of the Florida Current from the Straits of Florida to Cape Fear. Deep-Sea Res., Suppl., 16, 228-233.

Robinson, A. R., 1965: Oceanography. Research Frontiers in Fhid Dynamics, Chap. 17, New York, Interscience p. 738.

Stommel, H., 1965: The Gulf Stream. Berkeley, University of California Press, $248 \mathrm{pp}$.

-_, and A. B. Arons, 1960: On the abyssal circulation of the world ocean-II. An idealized model of circulation pattern and amplitude in oceanic basins. Deep-Sea Res., 6, 217-233.

Sverdrup, H. U., 1947: Wind-driven currents in a baroclinic ocean, with application to the equatorial currents of the eastern Pacific. Proc. Natl. Acad. Sci., 23, 318-326.

Veronis, G., 1966: Wind-driven ocean circulation-Part 2. Numerical solutions to the non-linear problem. Deep-Sea Res., 13, 31-55.

- $1969:$ On theoretical models of the thermocline circulation. Deep-Sea Res., Suppl., 16, 301-323.

Welander, P., 1968: Wind-driven circulation in one- and twolayer oceans of variable depth. Tellus, 20, 1-15.

Worthington, L. V., 1965: On the shape of the Gulf Stream system. Trans. Amer. Geophys. Union, 46, 99.

Wyrtki, K., and K. Haberland, 1968: On the redistribution of heat in the North Pacific Ocean. J. Oceanogr. Soc. Japan, 24, $220-233$

Yoshida, K. 1965: A theoretical model on wind-induced density field in the oceans I. J. Oceanogr. Soc. Japan, 21, 16-35. 